\title{
Phage therapy: unexpected drawbacks to reach hospitals
}

\author{
Andrea Álvarez ${ }^{1}$, Lucía Fernández ${ }^{2,3}$, Beatriz Iglesias ${ }^{4}$, Javier Rodríguez ${ }^{4}$ Ana Rodríguez $z^{2,3}$ \\ \& Pilar García*,2,3 \\ ${ }^{1}$ Servicio de Medicina Interna, Hospital San Agustín, 33401 Avilés, Asturias, Spain \\ ${ }^{2}$ Instituto de Productos Lácteos de Asturias (IPLA-CSIC), Paseo Río Linares, 33300 Villaviciosa, Asturias, Spain \\ ${ }^{3}$ DairySafe Group, Instituto de Investigación Sanitaria del Principado de Asturias (ISPA), 33011 Oviedo, Asturias, Spain \\ ${ }^{4}$ Servicio de Microbiología, Hospital San Agustín, 33401 Avilés, Asturias, Spain \\ *Author for correspondence: pgarcía@ipla.csic.es
}

\section{" until recently, we did not give too much thought to antibiotic resistance, because it used to be relatively easy to find another suitable antibiotic when an older one stopped working”}

First draft submitted: 9 November 2019; Accepted for publication: 6 January 2020; Published online: 30 January 2020

Keywords: antibiotic resistance $\bullet$ hospitals $\bullet$ infectious diseases $\bullet$ new antibiotics $\bullet$ phage therapy

The use of phages to kill pathogenic bacteria is an old practice to cure infectious diseases. The current antibiotic crisis has boosted phage therapy research, but its application in hospitals is still very limited. Here, we analyze the problems that need to be solved to go forward with this renewed therapy, hoping that it can be useful for patients suffering from an incurable infection. We would like to emphasize that phage therapy is right now at a crossroads. This may be similar to what happened a century ago with the arrival of antibiotics, but now we do not have such a good alternative. Therefore, let us not waste this opportunity, even if a radical change in our health strategy is necessary.

When a patient arrives at a hospital to be treated for a bacterial infectious disease, a process to confirm and identify the causative agent begins. Once the bacterium has been isolated, an antibiogram is performed, which will provide key information to guide the treatment. In an antibiogram, the bacterial sensitivity to a number of antibiotics [1] previously chosen by the hospital committee is tested. This decision takes into consideration local epidemiology (e.g., a bacterial resistance map is continuously updated) as well as National and International Guidelines, such as the WHO Global Action Plan on AMR [2].

Fortunately, there still is a suitable antibiotic for most infections (i.e., antibiograms usually show sensitivity for at least one of the tested antibiotics). Nevertheless, doctors more and more often have to resort to last generation antibiotics because the pathogenic bacterium is resistant to first choice antibiotics.

But what happens when the bacteria causing infection are not sensitive to the available antibiotics? When this occurs, doctors have the possibility to ask the Health Authorities for permission to apply a therapy under development, such as phage therapy, which is based on the use of bacteriophages (or phages) to treat infectious diseases. Bacteriophages were discovered a century ago and have, since then, provided valuable information about molecular biology, evolution and virulence, but have also been amazing tools for genomic engineering and disease diagnosis. In fact, treatment of bacterial infections was the first notable use of phages [3,4]. At that time, the knowledge about phage biology was very limited and their application as medicine was mostly empirical. This scenario paved the way for their abandonment when antibiotics were discovered.

Nowadays, phage therapy can be applied under a treatment option named Compassionate Use (specified in the Helsinki Declaration of Ethical Principles for Medical Research Involving Human Subjects), which allows patients suffering from a disease with no efficient standard therapy the use of other medicines, unauthorized or only available in other countries. However, prior to the approval of a therapeutic compound for Compassionate Use, the European Medicines Agency requires that the treatment be undergoing clinical trials or have entered the marketing-authorization application process (although legal requirements vary between countries) [5]. Due to the 
fact that phages do not fulfill any of these requirements, compassionate use is only available in countries with a specific regulation such as Belgium, France and the USA, as well as in countries where phage therapy has been maintained from the beginning (the 1930s) such as Poland, Georgia and Russia. In the rest of the world, access to phage therapy is hindered by the lack of results from clinical trials. To date, only one clinical trial has been carried out in the European Union (PhagoBurn), which was intended for testing the efficacy and tolerability of a cocktail of lytic anti-Pseudomonas aeruginosa bacteriophages to treat burn wounds infections. Nonetheless, it is worth mentioning that several clinical trials are currently ongoing in different countries [6], although their results have still not been released. Given the obvious need for clinical trials, why have they not been performed yet? There are three main obstacles to carry out a clinical trial with bacteriophages:

First, the economic cost which is usually supported by governments or pharmaceutical companies, the latter remaining skeptical due to the confusing legal framework along with the need to customize treatment for each patient. By contrast, antibiotics are produced at a large scale and have a wide bacterial spectrum, which has made them very attractive for pharmaceutical companies.

Second, it is necessary to invest time and effort to get the authorization from the relevant country's competent authority. Many of these difficulties are derived from the special nature of phages and their differences with classical antimicrobials. For example, unique traits of bacteriophages, such as their ability to propagate in bacteria and potentially transfer genetic material or their high host specificity, require different handling and the development of a new legal framework [7].

Third, it is quite difficult to recruit volunteers who meet the requirements of these clinical trials (multi-resistant infectious disease, physician and patient consent, availability of properly purified phage preparations). In Europe, the European Medicines Agency is responsible for the evaluation of new treatments through expert committees. Their decision goes to the European Commission, who will then authorize or deny marketing of such medicine. However, the pharmaceutical legislation applicable to antibiotics is not appropriate for phages, which would be considered as part of advanced therapy medicinal products. Therefore, proper regulation of phage use should be implemented at the national and international levels [8].

Coming back to our patient waiting for a successful treatment, the next option is to travel to the previously mentioned countries where phage therapy is possible (Queen Astrid Military Hospital, Belgium; Hospices Civils de Lyon, France; Phage Therapy Unit, Poland; Phage Therapy Center, Georgia; Center for Innovative Phage Applications and Therapeutics, CA, USA). If this is the case, some considerations have to be taken into account. For instance, the high specificity of phages for their host bacteria is a very valuable characteristic, as only target bacteria will be removed after treatment, while beneficial microbiota will remain unaltered. Nevertheless, this fact requires the selection of infective phages for each strain from each patient. Phage production centers usually have large phage collections, making it easy to find a suitable one, but it remains necessary for the patient's strain to be isolated and tested before the treatment (e.g., by performing a phagogram test, which would be the phage equivalent of an antibiogram test). Once identified, the proper phage or phages that infect and lyse the pathogenic bacterium [9], they have to be propagated and purified (in an authorized laboratory or company) following the recommendations of the European Pharmacopoeia, or the Belgian Pharmacopoeia in case of the Magistral Phage preparations. Indeed, these magistral preparations can contain nonauthorized ingredients as long as they are accompanied by a certificate of analysis issued by a Belgian Approved Laboratory [10]. In the last 2 years, external requests for phage therapy in phage therapy centers have increased significantly [11] and several successful cases have already been reported [12-15].

Antibiotics have saved countless human lives and have revolutionized modern medicine. After their discovery, these antibacterial weapons quickly improved the health of the population in developed countries, becoming the panacea for infectious diseases, which were very abundant at that time. But this honeymoon was actually very short. Indeed, the first penicillin-resistant bacterium was isolated in 1945. However, until recently, we did not give too much thought to antibiotic resistance, because it used to be relatively easy to find another suitable antibiotic when an older one stopped working. This period of optimism is now almost over. As a consequence of the current state of the antibiotic resistance epidemic, there has been a paradigm shift. Physicians have become much more careful and initially choose the least powerful antibiotic to which the bacterium is sensitive. Most hospitals now have a surveillance group to implement a rational use of the available antibiotics. In this regard, WHO updates every 2 years a Model Lists of Essential Medicines [16], where antibiotics are classified (AWaRe classification) into three different groups. Recommendation of their priority for a proper use is also displayed (access, antibiotics for first or second choice; watch, only used for specific treatments; and reserve, last resort antibiotics only used against multi-resistant bacteria). 
It seems clear that the surveillance of multi-resistant bacteria is key to manage the rational use of antibiotics and to reduce the future incidence of resistance. Indeed, the implementation of the AMR Global Action Plan is already being progressively carried out by the different countries and implies greater control in the use of antibiotics and the surveillance of antimicrobial resistance, among other strategies. Specifically, the Global Antimicrobial Surveillance System (GLASS) reveals widespread occurrence of antibiotic resistance among 500,000 people with suspected bacterial infections across 22 countries [17].

On the other hand, the current antibiotic crisis has boosted phage therapy research in the last decade, yielding many promising results [18]. However, despite the successful data from animal models of infection, the route to actually put this strategy into practice at hospitals seems to be more complex than initially thought. The truth is that, despite the urgent need for alternatives to antibiotics, the use of phage-derived products in hospitals is still very limited and physicians sometimes feel helpless in front of a patient suffering from an incurable infection. Indeed, about 33,000 people die each year in the European Union as a consequence of multidrug-resistant bacteria causing infections [19].

In view of these results, researchers are pushing the health authorities to make changes to the current regulations to facilitate the transfer of their results to pharmaceutical companies [8]. Meanwhile, patients are suffering all kinds of inconveniences in a race against time to fight bacterial infections.

\section{Conclusion}

The global antibiotic crisis is questioning the current strategy to combat infections mainly based on antibiotics (therapeutics) and vaccines (prophylaxis). In this regard, important research is being done into antimicrobial alternatives such as phage therapy. However, promising results in the use of phages to treat human infections are limited by the lack of investment, little interest by pharmaceutical companies and law regulations. A change in the current structure of medicine toward a personalized medicine might facilitate the arrival of phage therapy to Western countries.

\section{Future perspective}

The foreseeable advance of biotechnology and biomedicine over the next few years will allow solving the current problems of phage therapy, so that it is expected that phages will be available as a therapeutic alternative in hospitals. In this regard, we should have large phage collections (perfectly characterized) against most infectious bacteria as well as a rapid system for identification of phages infecting those bacteria isolated from patients. Finally, we should also have suitable stocks of these purified phages in order to easily prepare the suitable cocktail to further treat a particular patient.

\section{Author contributions}

All authors participated in the writing of this manuscript.

\section{Acknowledgments}

P Garcia dedicates this manuscript to NV, for her courage in the fight against an infectious disease.

\section{Financial \& competing interests disclosure}

This study was funded by grants AGL2015-65673-R (MINECO/FEDER/EU, Spain), EU ANIWHA ERA-NET (BLAAT ID: 67)/PCIN2017-001 (AEI/FEDER/EU, Spain), Proyecto Intramural CSIC 201770E016, IDI/2018/000119 (Asturias Innovation 2018-2020, Principado de Asturias, Spain) and FEDER/EU. P Garcia and A Rodríguez are members of the bacteriophage network FAGOMA and the FWO Vlaanderen funded 'Phagebiotics' research community (WO.016.14). The authors have no other relevant affiliations or financial involvement with any organization or entity with a financial interest in or financial conflict with the subject matter or materials discussed in the manuscript apart from those disclosed.

No writing assistance was utilized in the production of this manuscript.

\section{References}

Papers of special note have been highlighted as: • of interest; $\bullet \bullet$ of considerable interest

1. Tascini C, Sozio E, Viaggi B, Meini S. Reading and understanding an antibiogram. Ital. J. Med. 10(4), 289-300 (2016).

- Complete description of parameters to consider in the design and interpretation of antibiograms. 
2. WHO. Global action plan on antimicrobial resistance. World Health Organization (2015). www.who.int/antimicrobial-resistance/global-action-plan/en/

-• A summary of all actions proposed by World Health Organization to tackle antimicrobial resistance in the world.

3. Sulakvelidze A, Morris JG Jr. Bacteriophages as therapeutic agents. Ann. Med. 33(8), 507-509 (2001).

4. Cisek AA, Dabrowska I, Gregorczyk KP, Wyzewski Z. Phage therapy in bacterial infections treatment: one hundred years after the discovery of bacteriophages. Curr. Microbiol. 74(2), 277-283 (2017).

5. Borysowski J, Ehni HJ, Gorski A. Ethics review in compassionate use. BMC Med. 15, 136 (2017).

6. U.S. National Library of Medicine. Listing of Clinical trials currently ongoing (2019). www.clinicaltrials.gov/ct2/results? recrs=\&cond $=\&$ term $=$ phage\&cntry=\&state $=\&$ city $=\&$ dist $=$

7. Cooper CJ, Khan Mirzaei M, Nilsson AS. Adapting drug approval pathways for bacteriophage-based therapeutics. Front. Microbiol. 7 , 1209 (2016).

- A detailed description about the state-of-the-art in legal framework for bacteriophages and possible alternative pathways for the approval of phage therapy.

8. Fauconnier A. Phage therapy regulation: from night to dawn. Viruses. 11(4), 352 (2019).

9. Fernandez L, Gutierrez D, Garcia P, Rodriguez A. The perfect bacteriophage for therapeutic applications-a quick guide. Antibiotics (Basel) 8(3), 126 (2019).

- Detailed description for the selection of bacteriophages to be used as antimicrobials.

10. Pirnay JP, Merabishvili M, Van Raemdonck H, De Vos D, Verbeken G. Bacteriophage production in compliance with regulatory requirements. Methods Mol. Biol. 1693, 233-252 (2018).

11. Djebara S, Maussen C, De Vos D et al. Processing phage therapy requests in a Brussels Military Hospital: lessons identified. Viruses 11(3), 265 (2019).

12. Dedrick RM, Guerrero-Bustamante CA, Garlena RA et al. Engineered bacteriophages for treatment of a patient with a disseminated drug-resistant Mycobacterium abscessus. Nat. Med. 25(5), 730-733 (2019).

-. First case report about a person treated and cured with a genetically modified bacteriophage.

13. Aslam S, Courtwright AM, Koval C et al. Early clinical experience of bacteriophage therapy in 3 lung transplant recipients. Am. J. Transplant. 19(9), 2631-2639 (2019).

14. Ferry T, Boucher F, Fevre C et al. Innovations for the treatment of a complex bone and joint infection due to XDR Pseudomonas aeruginosa including local application of a selected cocktail of bacteriophages. J. Antimicrob. Chemother. 73(10), 2901-2903 (2018).

15. Onsea J, Soentjens P, Djebara S et al. Bacteriophage application for difficult-to-treat musculoskeletal infections: development of a standardized multidisciplinary treatment protocol. Viruses. 11(10), 891 (2019).

16. World Health Organization (WHO). Model Lists of Essential Medicines (2019). www.who.int/medicines/publications/essentialmedicines/en/

17. World Health Organization (WHO). Global Antimicrobial Surveillance System (GLASS) (2015). www.who.int/glass/en/

18. Romero-Calle D, Guimaraes Benevides R, Goes-Neto A, Billington C. Bacteriophages as alternatives to antibiotics in clinical care. Antibiotics (Basel) 8(3), 138 (2019).

19. Cassini A, Hogberg LD, Plachouras D et al. Attributable deaths and disability-adjusted life-years caused by infections with antibiotic-resistant bacteria in the EU and the European Economic Area in 2015: a population-level modelling analysis. Lancet Infect. Dis. 19(1), 56-66 (2019).

-. Description of the current scenario in Europe regarding the impact of antibiotic-resistant bacteria in population. 